\title{
Predicting liver cytosol stability of small molecules
}

\author{
Pranav Shah ${ }^{\dagger}$, Vishal B. Siramshetty ${ }^{\dagger}$, Alexey V. Zakharov, Noel T. Southall, Xin Xu and Dac-Trung Nguyen ${ }^{*}$
}

\begin{abstract}
Over the last few decades, chemists have become skilled at designing compounds that avoid cytochrome P (CYP) 450 mediated metabolism. Typical screening assays are performed in liver microsomal fractions and it is possible to overlook the contribution of cytosolic enzymes until much later in the drug discovery process. Few data exist on cytosolic enzyme-mediated metabolism and no reliable tools are available to chemists to help design away from such liabilities. In this study, we screened 1450 compounds for liver cytosol-mediated metabolic stability and extracted transformation rules that might help medicinal chemists in optimizing compounds with these liabilities. In vitro half-life data were collected by performing in-house experiments in mouse (CD-1 male) and human (mixed gender) cytosol fractions. Matched molecular pairs analysis was performed in conjunction with qualitative-structure activity relationship modeling to identify chemical structure transformations affecting cytosolic stability. The transformation rules were prospectively validated on the test set. In addition, selected rules were validated on a diverse chemical library and the resulting pairs were experimentally tested to confirm whether the identified transformations could be generalized. The validation results, comprising nearly 250 library compounds and corresponding half-life data, are made publicly available. The datasets were also used to generate in silico classification models, based on different molecular descriptors and machine learning methods, to predict cytosol-mediated liabilities. To the best of our knowledge, this is the first systematic in silico effort to address cytosolic enzyme-mediated liabilities.
\end{abstract}

Keywords: Xenobiotic metabolism, Cytosol stability, Matched molecular pairs, Qualitative-structure activity relationship, Machine learning

\section{Introduction}

Over the last few decades, chemists have become increasingly proficient at designing compounds that are able to circumvent cytochrome P (CYP) 450 mediated clearance. This has led to the emergence of other drug metabolizing enzymes such as esterases, aldehyde oxidase, etc. as significant contributors to drug clearance [1]. Typical screening assays are performed in liver microsomal fractions as they contain the CYP450 enzymes and it is possible to overlook the contribution

\footnotetext{
*Correspondence: nguyenda@mail.nih.gov

'Pranav Shah and Vishal B. Siramshetty contributed equally to this work National Center for Advancing Translational Sciences (NCATS), National Institutes of Health (NIH), 9800 Medical Center Drive, Rockville, MD 20850, USA
}

of non-microsomal clearance until later stages of the drug discovery process. In 2008, the development of compound SGX523 was discontinued after reports of dose-limiting renal toxicity [2]. Investigational New Drug-enabling studies for SGX523 were performed in dogs and rats since no significant differences were seen in rat, dog, monkey and human microsomal clearance profiles. It was later found that the toxicity occurred due to the accumulation of the aldehyde oxidase (AO)mediated insoluble toxic metabolite in the kidneys [2, $3]$. In the case of compound FK3453, clinical trials had to be terminated due to the abnormally low levels of parent compound in human plasma [4]. The hepatic availability in human was calculated by in vitro-in vivo scaling using in vitro intrinsic clearance data from liver microsomes and in vivo intrinsic clearance data

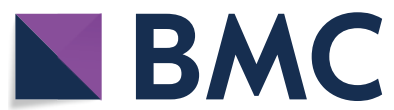

(c) The Author(s) 2020. This article is licensed under a Creative Commons Attribution 4.0 International License, which permits use, sharing, adaptation, distribution and reproduction in any medium or format, as long as you give appropriate credit to the original author(s) and the source, provide a link to the Creative Commons licence, and indicate if changes were made. The images or other third party material in this article are included in the article's Creative Commons licence, unless indicated otherwise in a credit line to the material. If material is not included in the article's Creative Commons licence and your intended use is not permitted by statutory regulation or exceeds the permitted use, you will need to obtain permission directly from the copyright holder. To view a copy of this licence, visit http://creativeco mmons.org/licenses/by/4.0/. The Creative Commons Public Domain Dedication waiver (http://creativecommons.org/publicdomain/ zero/1.0/) applies to the data made available in this article, unless otherwise stated in a credit line to the data. 
from rats and dogs. AO-mediated metabolism was found responsible for the low exposure of FK3453 in humans [4]. These examples highlight the importance of understanding the metabolism of new drug candidates in systems that contain the full array of metabolizing enzymes.

Several well-known structure modification strategies exist to guide the medicinal chemist through CYP-mediated instabilities [5, 6]. However, strategies to attenuate non-CYP-mediated liabilities are sparsely reported in the literature [7-10]. A few in-house projects at the National Center for Advancing Translational Sciences (NCATS) were recently discovered to have cytosol-mediated metabolic liabilities. We generated half-life $\left(\mathrm{t}_{1 / 2}\right)$ data for 1450 compounds using mouse and human cytosol fractions. The aim of this study is to explore these datasets and provide chemists with guidelines which can be used to rationally modulate compounds and attenuate cytosol-mediated metabolic instabilities. To achieve this, we performed matched molecular pairs analysis (MMPA) on the stability data measured in mouse and human cytosol fractions. This concept is widely applied in medicinal chemistry due to the possibility to associate defined structural transformations between compound pairs with corresponding changes in molecular properties [11-13]. Thus, MMPA allows us to extract inherent medicinal chemistry knowledge from these datasets and helps understand the impact of important chemical structure modifications on cytosolic stability [14]. The extracted rules were validated on an in-house small molecule library and their general tendency to improve cytosolic stability was experimentally validated. A detailed analysis of the structure-metabolism data revealed certain transformations that demonstrated opposite effects on the stability of compounds in human and mouse cytosol fractions. In addition, we present in silico classification models, based on by far the largest dataset reported in the literature, that will help facilitate identification of compounds with potential cytosol-mediated metabolic liabilities.

\section{Methods and data \\ Metabolic stability assay}

Mixed gender human (H0610.C) and male CD-1 mouse liver cytosol fractions (M1000.C) were purchased from Sekisui XenoTech, LLC (Kansas City, USA). We chose the substrate depletion method to determine in vitro $t_{1 / 2}$. Our assay (384-well format) consisted of two parts; a robotic system for incubation and sample clean up and an integrated LC/MS method to calculate the percent remaining of parent compound. Briefly, each reaction mixture $(110 \mu \mathrm{L})$ consisted of a test compound $(1 \mu \mathrm{M})$ and either human or mouse cytosol fractions $(2 \mathrm{mg} / \mathrm{mL})$ in phosphate buffer $(100 \mathrm{mM})$ at $\mathrm{pH}$ 7.4. Samples were incubated in 384-well plates at $37^{\circ} \mathrm{C}$ for $0,5,10,15,30$ and $60 \mathrm{~min}$. Sample analysis and $t_{1 / 2}$ calculations were performed using a previously described method [15]. Using this high-throughput method, we generated $t_{1 / 2}$ data for 1450 compounds in mouse and human cytosol fractions. A majority of these compounds were part of three on-going in-house projects. A set of control compounds for low and high cytosolic stability were routinely run in each plate. The reproducibility data for exemplary control compounds used in the assay are presented in Table 1. The minimum significant ratio (MSR) for famciclovir (human and mouse) as well as carbazeran (human) were found to be below 3 which indicates the robustness of the assay.

\section{Training data}

The set of 1450 compounds tested in cytosolic stability assay (human and mouse) is considered as the training set. For the purpose of this study, we distinguish unstable compounds from stable compounds using the following criteria: $\mathrm{t}_{1 / 2} \leq 30 \mathrm{~min}$ - unstable; $\mathrm{t}_{1 / 2}>30 \mathrm{~min}$-stable. The chemical structures of the compounds were standardized, and the normalized structures were used to generate standard InChIKey notations. The steps involved

Table 1 Reproducibility across different experiments

\begin{tabular}{llll}
\hline Compound & \# Repeats $^{\mathbf{a}}$ & $\begin{array}{l}\mathbf{t}_{\mathbf{1 / 2}} \text { (mean }^{(\text {SD)/category }} \\
\text { (Mouse cytosol) }^{\mathbf{b}}\end{array}$ & $\begin{array}{l}\mathbf{t}_{\mathbf{1 / 2}} \text { (mean } \pm \text { SD)/category } \\
\text { (Human cytosol) }^{\mathbf{b}}\end{array}$ \\
\hline Buspirone & 17 & $>120 \mathrm{~min} / \mathrm{High}$ & $>120 \mathrm{~min} / \mathrm{High}$ \\
Antipyrine & 17 & $>120 \mathrm{~min} / \mathrm{High}$ & $>120 \mathrm{~min} / \mathrm{High}$ \\
Famciclovir & 17 & $9.5 \pm 3.2 \mathrm{~min} / \mathrm{Low}$ & $10.0 \pm 3.1 \mathrm{~min} / \mathrm{Low}$ \\
Carbazeran & 17 & $>120 \mathrm{~min} / \mathrm{High}$ & $3.5 \pm 1.8 \mathrm{~min} / \mathrm{Low}$ \\
\hline
\end{tabular}

\footnotetext{
${ }^{a} t_{1 / 2}$ values of the control compounds across 17 plates were measured by UHPLC/HRMS. $t_{1 / 2}$ categories: $<10 \mathrm{~min}$ : low; $>30 \mathrm{~min}$ : high
}

${ }^{b}$ No SD values provided if all replicate $\mathrm{t}_{1 / 2}$ values exceeded $120 \mathrm{~min}$ 
in standardization of chemical structures are detailed in Additional file 1: Table S1. Entries sharing the same standard InChIKey were marked as duplicates and the compound was retained only if all corresponding entries belonged to the same class (i.e., stable or unstable). Finally, the training sets from both human and mouse cytosol fractions consisted of 1230 unique compounds.

\section{Matched molecular pairs analysis setup}

A matched molecular pair can be defined as a pair of compounds that differ only at a single site through a well-defined structural transformation that is associated with a relative change in a property value $[11,13,16]$. In this study, MMPA was performed using the open-source KNIME Analytics Platform (version 3.7) [17] that facilitates automatization of data integration and analysis for a range of cheminformatics tasks. The Vernalis KNIME nodes [18], that reproduce the Hussain and Rea algorithm [16], were used to perform MMPA in addition to different RDKit [19] community nodes. The steps involved are molecule fragmentation, identification of matched pairs and compilation of unique transformation rules. In order to prospectively validate the transformations, training dataset was further divided into two parts, i.e., training and test set, at a ratio of 70:30 in a random manner. The same random seed was used for both human and mouse data so that their training and test set compositions are similar. The KNIME workflow for MMPA can be found in the supporting material (Additional file 2).

\section{Cytosolic stability prediction model}

Next, we sought to develop in silico classifiers based on the human cytosol stability data to facilitate identification of potential substrates during the lead optimization phase. Since the mouse data was found to be heavily skewed towards the stable class, we decided to build a predictive model based on the human dataset. The same dataset used for the generation of MMP rules was employed for this purpose. Binary classifiers were built using Random Forests (RF) [20], Support Vector Machines (SVM) [21, 22] and BayesNet [23] approaches. RF and SVM algorithms were chosen on the basis of their popularity in predicting molecular properties [24]. BayesNet node, made available in KNIME by WEKA, facilitates learning using different Bayes Network learning algorithms. The method was previously employed by Zakharov et al. [25] in predicting metabolic stability of compounds in human liver microsomes and was reported to be among those methods that provided higher sensitivity in predicting unstable compounds. Three sets of molecular descriptors were independently used as features: two-dimensional (2D) descriptors available from the Molecular Operating Environment (MOE; Chemical
Computing Group ULC.) [26]; 2D descriptors calculated from the RDKit Descriptor Calculation node in KNIME; and QNA descriptors $[27,28]$. The descriptors from MOE and RDKit were normalized, a pair-wise correlation analysis was performed, and the most-correlated $\left(\mathrm{r}^{2}>0.9\right)$ and constant value descriptors were removed, which left 119 MOE descriptors (out of 206) and 80 RDKit descriptors (out of 119). A total of 120 QNA descriptors include 10 whole molecule 2D descriptors and 110 QNA descriptors accounting for ionization potential and electronic affinity of individual atoms based on a connectivity matrix representation [28]. The QNA descriptors were not subjected to correlation analysis and normalization due to their orthogonal nature but any constant-value descriptors were omitted. These descriptors were selected as they were previously reported to perform well in the prediction of $t_{1 / 2}$ values in human liver microsomes [25]. In addition to the models based on RF, SVM and BN, we built a deep learning model based on a directed message passing neural network recently proposed by Yang et al. [29] for molecular property prediction. It is a graph convolutional neural network (GCNN) that operates on graphs structures of molecules to construct a learned molecular representation. This model was selected on the basis of its competitive performance on a large number of datasets as compared to the publicly available benchmark from Wu et al. [30].

Since the training set was skewed towards the majority class (i.e., stable compounds), we generated balanced training sets by under-sampling the majority class. Two different methods were employed: diversity under-sampling and multiple under-sampling. In the first method, chemically diverse compounds were picked from the majority class using the RDKit diversity picker node which uses molecular fingerprints to pick an arbitrary number of diverse compounds. The number of compounds picked is equal to the number of compounds in the minority class (i.e., unstable compounds). In multiple under-sampling, majority class instances are randomly sampled to the size of minority class and this was repeated until each majority class instance has become part of the training set at least once. Average of the individual models that are based on distinct subsets of majority class were reported as the final performance values [31]. The models were evaluated using different performance metrics. An overview of these metrics can be found in Additional file 1: Table S5. A complete workflow that represents the methodology is shown in Fig. 1.

\section{MMP and QSAR model: external validation}

The transforms from the training sets were applied to the test sets in a prospective manner. Further, selected transformations from the training set were validated on 


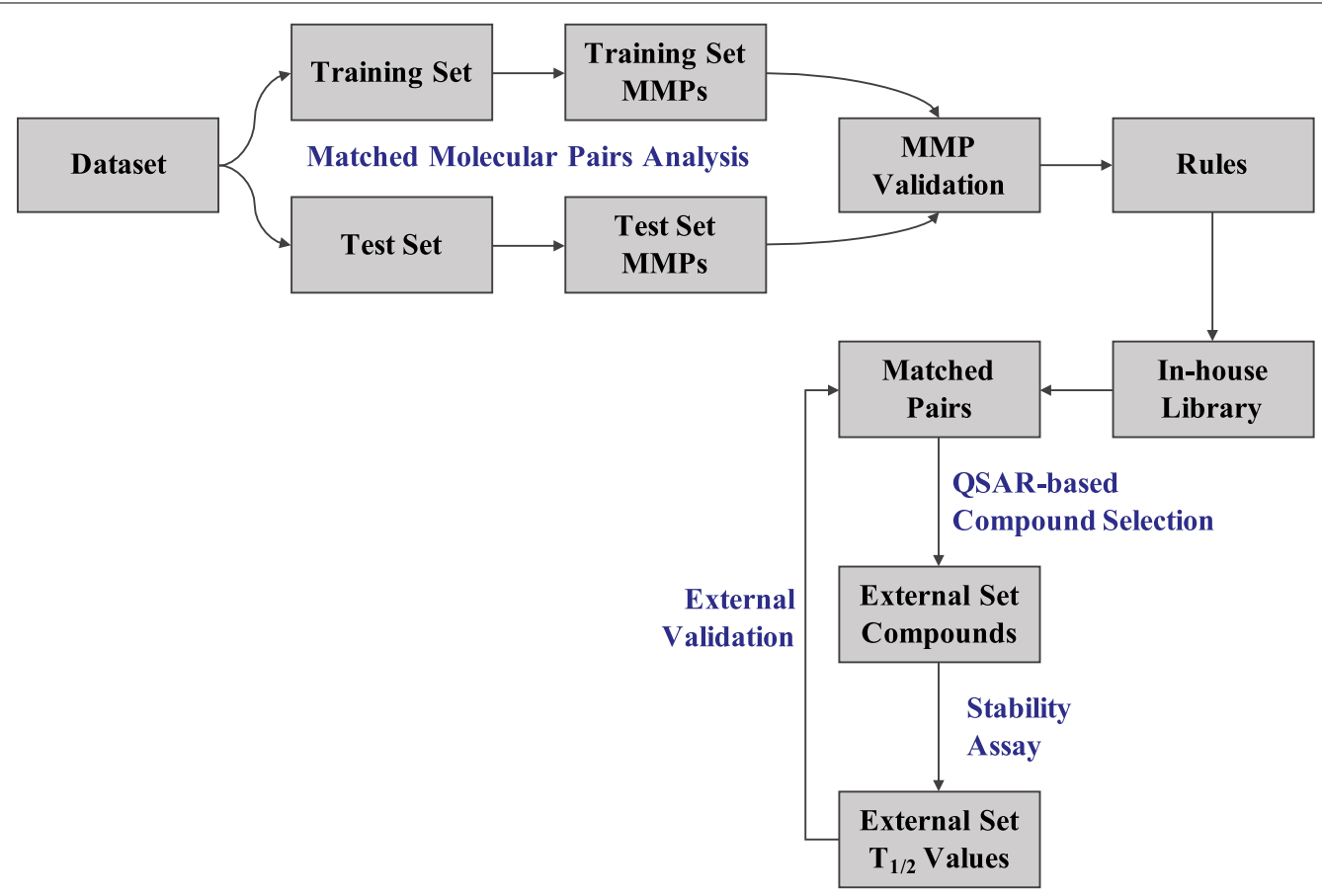

Fig. 1 A schematic representation of the study methodology

an independent external dataset (in-house library consisting of 96,358 compounds) using the 'RDKit Apply Transformations' node. The library comprises a large number of core scaffolds, representing 20 to $100 \mathrm{com}$ pounds per chemotype that facilitate optimization efforts in medicinal chemistry projects and therefore fits well with the scope of the study. The node takes a list of transformations and a set of molecules as input and provides an additional column containing the transformed molecules. We then checked if the transformed molecules were present in our library to generate a list of matched pairs that obey the rules derived from the training set. These independent compound pairs were tested in the same cytosolic stability assay to assess if the transforms improve cytosolic stability.

For human dataset, we obtained a large number of such pairs. In order to select a limited number of pairs for experimental validation, we picked those matched pairs in which the compound on left was predicted as unstable by the QSAR model based on training set. A total 160 compounds (80 matched pairs) were chosen for experimental validation. Once the stability data was generated (we could obtain data for only 155 compounds), we used the same set for validating the QSAR model. Therefore, this set of 155 compounds is considered as external validation set. For the mouse dataset, only 29 pairs (58 compounds) were found in the library and we tested all 29 pairs as part of the external validation.

\section{Results}

\section{Molecular properties and data distribution}

From the distribution of simple molecular properties such as $\log$ P, topological polar surface area (TPSA) and molecular weight (MW) for the training set compounds (Figs. 2, 3), a majority of compounds have $t_{1 / 2}$ values greater than 30 min in human and mouse cytosol fractions, belong in the 300-500 MW range, have TPSA $<100$ and $\log \mathrm{P}$ values ranging from 2.0 to 5.0. No direct correlation could be detected between the experimental $t_{1 / 2}$ values and the calculated molecular properties. Furthermore, significant differences in the distribution of $t_{1 / 2}$ values were observed between the mouse and human datasets. Of the 1230 compounds, 313 (25.4\%) compounds were classified as unstable $\left(\mathrm{t}_{1 / 2}<30 \mathrm{~min}\right)$ in human dataset as compared to $128(10.4 \%)$ in the mouse dataset. 92 compounds were found to be unstable in both mouse and human datasets (Fig. 2 a, b).

\section{MMPs from training data (prospective validation)}

Compounds from the training set were processed in the MMP workflow. Prospective training set and test set from both species were treated exactly the same. Unique transformations were generated from the training and test sets, followed by identification of a list of transformations common to both these sets. This serves as prospective validation of the list of common transforms as though they were selected from the training set and applied on 

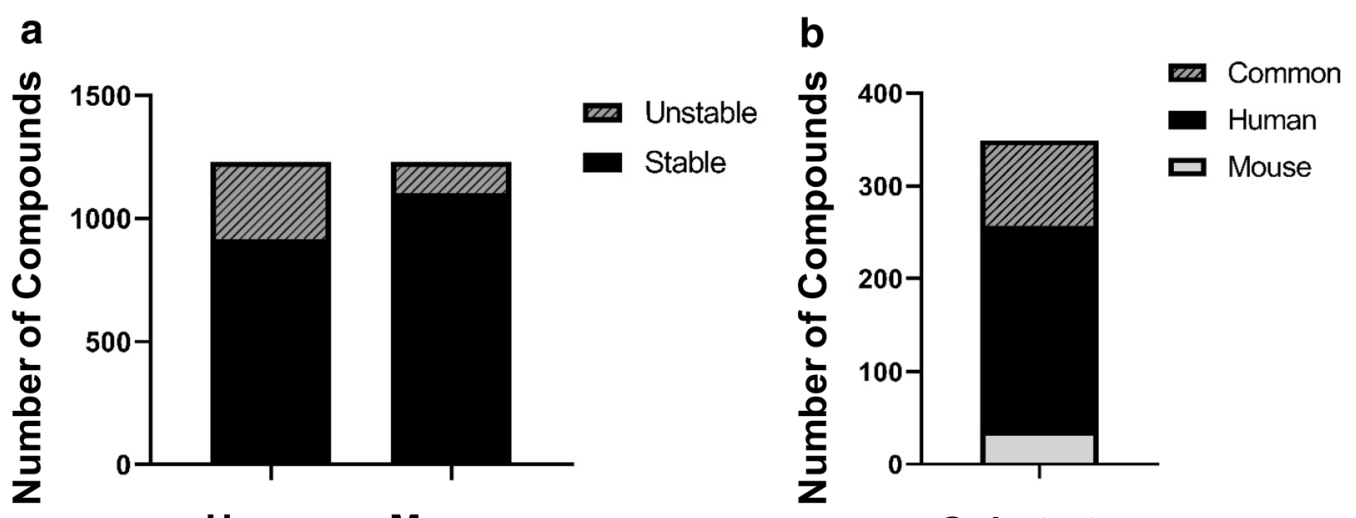

Human Mouse

Substrates

Fig. 2 a Distribution of training set compounds from human and mouse datasets. b Overlap of substrates across datasets
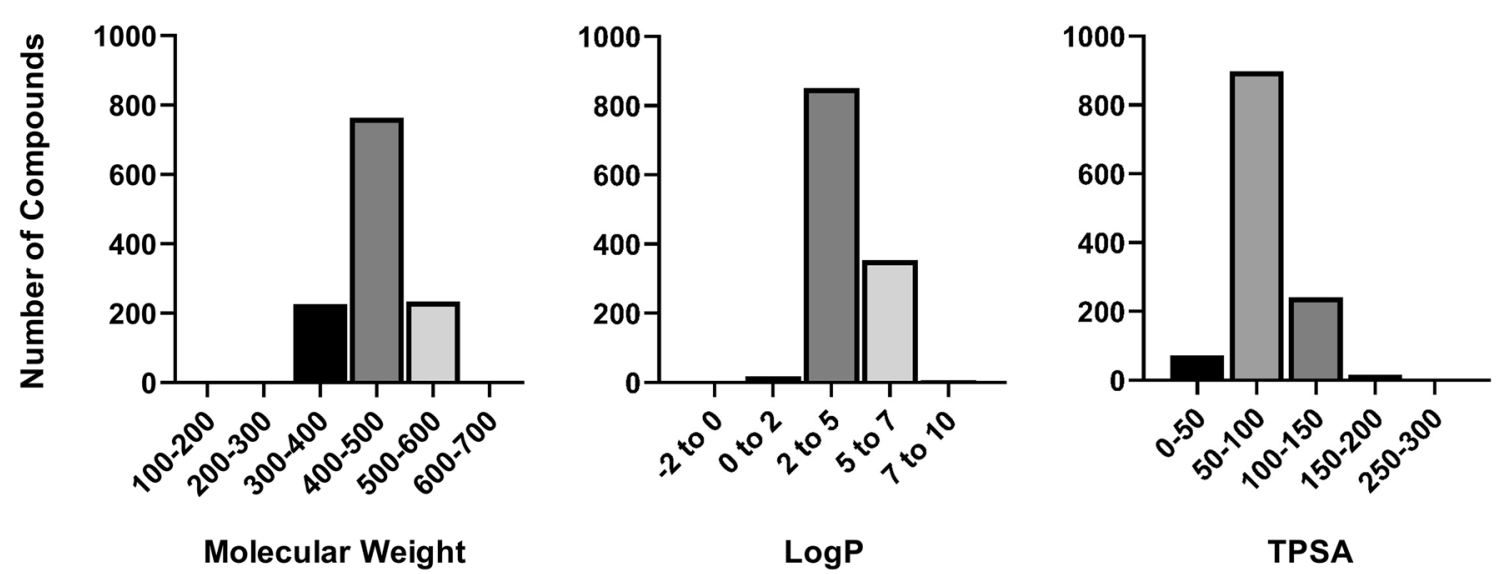

Fig. 3 Distribution of training set compounds on the basis of molecular properties

the test set compounds for validation. The training sets comprised of 1100 (human dataset) and 1105 (mouse dataset) compounds which represent $70 \%$ of the corresponding individual training sets. The remaining $30 \%$ portion served as a prospective test set. A total of 5292 unique transforms were identified from the two training sets. The overlap of training set transforms from human and mouse data, and their frequencies are presented in Figs. 4 and 5. It is clear that the human training set provided more transformations. However, the difference was less pronounced when only transforms with at least two or three examples each were considered. Similarly, the number of transforms in common decreased with the frequency criteria. Overall, the transforms from human dataset were more frequently found as compared to the transforms from mouse dataset. Since the compounds demonstrated different behaviors in mouse and human cytosol fractions, the number of transforms from both these datasets differ.
As part of the validation, 94 human transforms were applied on 46 test set compounds. In $50 \%$ of the cases, the $t_{1 / 2}$ increased by at least 1.5 -fold. Only 12 out of the 46 compounds were unstable. The transformations could successfully transform nine compounds from unstable to stable. Similarly, 44 transforms from mouse dataset were applied on 20 prospective test set compounds. In at least $50 \%$ of the cases, the $t_{1 / 2}$ could be increased by at least 1.5 times and five out of the seven unstable compounds were successfully transformed into stable analogues. The top 10 transformations form both datasets that could successfully transform unstable compounds to stable are listed in Additional file 1: Tables S2, S3.

\section{Species differences}

It is interesting to note that the mouse dataset contains more stable compounds than the human dataset. As pointed out earlier, clinical development of some drug candidates were terminated due to their unfavorable 

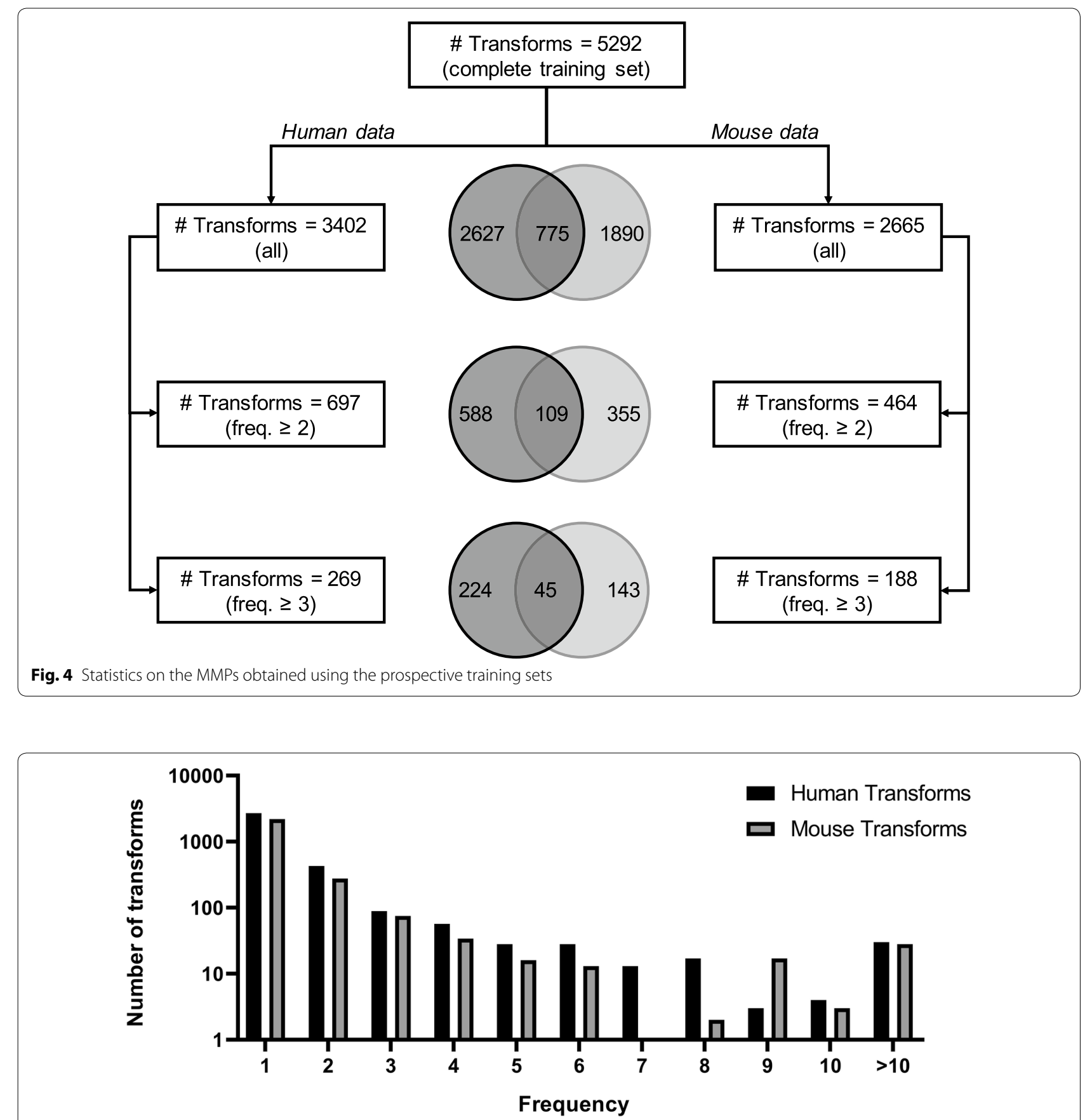

Fig. 5 Distribution of transforms from human and mouse datasets based on frequency

metabolic profiles in human that were not predicted during preclinical studies [32, 33]. This could explain why the dataset is more skewed toward the 'stable' class in the mouse dataset. Further investigation led to the identification of some transforms that showed opposite effects in human and mouse (see Table 2). Here, we consider only those examples in which the compound pairs involved in the transformation are identical, i.e., the same compound pair demonstrates a positive shift in mouse and a negative shift in human or vice versa. This analysis was beyond the scope of the study and we plan to pursue this further as part of a future study. 
Table 2 MMP transforms that demonstrated opposite behavior in human and mouse cytosol fractions

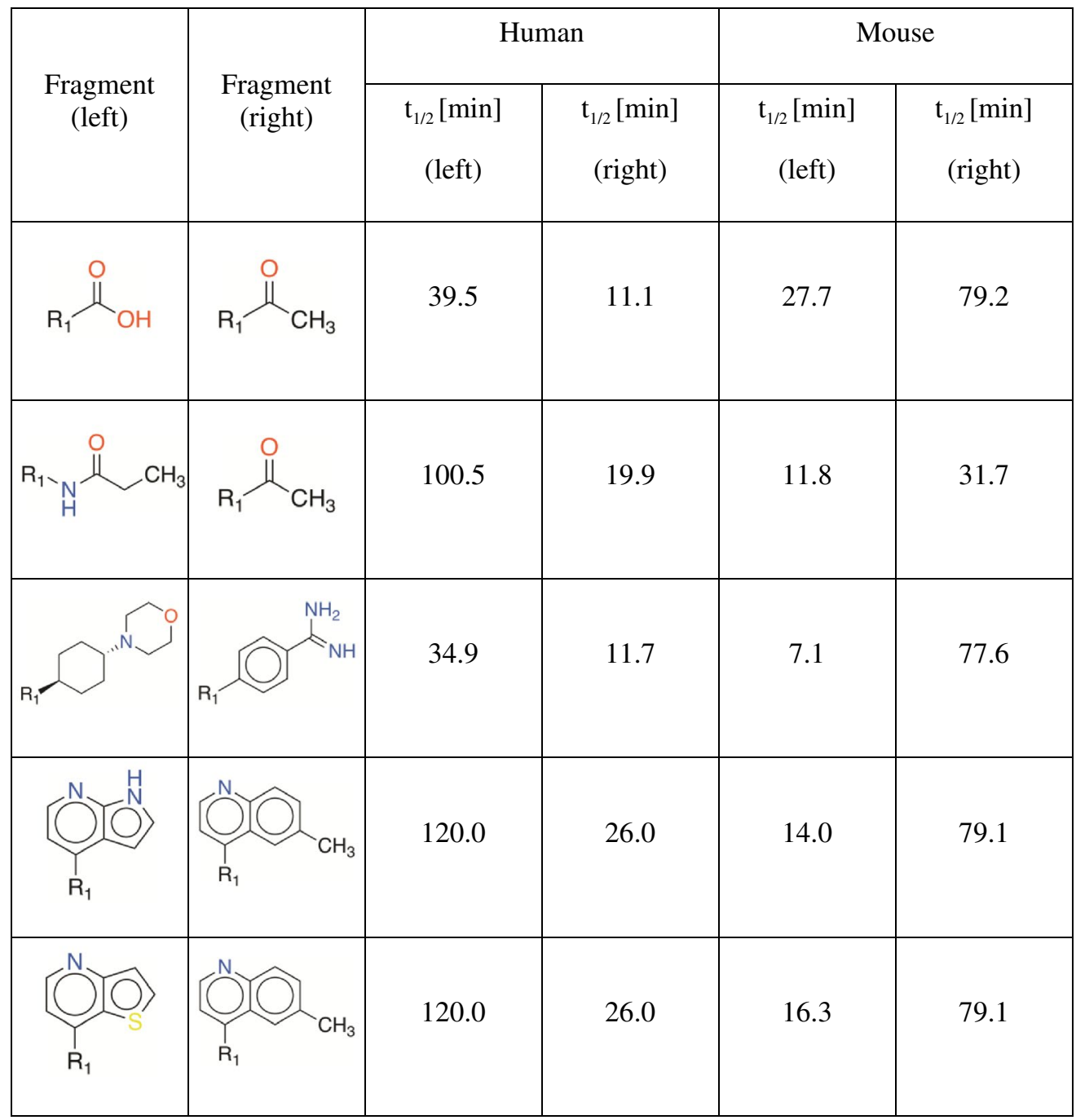

\section{Cytosolic stability prediction models}

The primary reason behind building a QSAR model based on this dataset was to prioritize the compound pairs that resulted from application of MMP rules identified in the first part of the analysis. However, we pursued it further to explore if the dataset in hand could be of any use to develop computational models to identify potentially unstable compounds in cytosolic fractions. During cross-validation (see Table 3), SVM-based classifiers performed on average better as compared to the classifiers based on RF and $\mathrm{BN}$ approaches. Although the AUC and BACC values did not differ significantly between SVM and RF models, SVM tended to provide higher Sensitivity (i.e., the ability to correctly predict unstable compounds) while RF provided higher Specificity. Although the BN models provided
Table 3 Cross-validation results

\begin{tabular}{llllll}
\hline Descriptor & Method $^{\mathbf{a}}$ & AUC-ROC & BACC & Sensitivity & Specificity \\
\hline MOE & BayesNet- & 0.81 & 0.76 & 0.72 & 0.79 \\
& DU & & & & \\
& RF-MU & 0.86 & 0.78 & 0.79 & 0.76 \\
& SVM-DU & 0.85 & 0.77 & 0.79 & 0.76 \\
RDKit & BayesNet & 0.79 & 0.73 & 0.61 & 0.85 \\
& RF-MU & 0.86 & 0.78 & 0.78 & 0.78 \\
& SVM-DU & 0.83 & 0.77 & 0.83 & 0.72 \\
QNA & BayesNet- & 0.75 & 0.69 & 0.62 & 0.75 \\
& MU & & & & \\
& RF-MU & 0.83 & 0.75 & 0.72 & 0.78 \\
& SVM-DU & 0.85 & 0.80 & 0.80 & 0.80 \\
\hline
\end{tabular}

a DU and MU stand for diversity under-sampling and multiple under-sampling, respectively 
inferior BACC values in general, they provided a balance between Sensitivity and Specificity. No significant differences in performance could be detected in terms of the different descriptors. As described earlier, we assessed the influence of under-sampling the majority class on model performance. In case of RF classifiers, the training set based on multiple under-sampling clearly provided better BACC with Sensitivity being the major contributor. While no consistent trend could be detected for BN classifiers, the SVM classifiers demonstrated an improvement with both down-sampling approaches. RF and SVM models banked more from under-sampling the majority class. The best performing model was an SVM classifier based on diversity under-sampling method and QNA descriptors with BACC, Sensitivity and Specificity of 0.8 each and an AUC of 0.85 .

\section{QSAR validation}

In external validation, surprisingly, the SVM classifiers demonstrated a poor performance. BayesNet classifiers always provided the best performance based on the original training set. In majority cases, diversity undersampling resulted in a poor performance due to a drop in Sensitivity. RF classifiers based on MOE descriptors were least affected by this. Although BN classifiers provided higher Sensitivity values, the highest BACC was achieved by the RF classifier based on MOE descriptors and original training set. Again, no significant differences in performance could be found between the models on the basis of different descriptors. However, in case of SVM and BN, QNA descriptors provided a better balance between Sensitivity and Specificity. When using MOE or RDKit descriptors, these models tended to predict all compounds as stable. In Tables 3 and 4, for each descriptor and method, only the performance of the best model (out of the three models: no sampling, diversity under-sampling, multiple under-sampling) was provided. The complete results for cross-validation and external validation are provided in Additional file 1: Table S6 and

\section{Table 4 External validation results}

\begin{tabular}{llllll}
\hline Descriptor & Method & AUC-ROC & BACC & Sensitivity & Specificity \\
\hline MOE & BayesNet & 0.62 & 0.60 & 0.75 & 0.45 \\
& RF & 0.73 & 0.64 & 0.63 & 0.66 \\
& SVM-MU & 0.51 & 0.56 & 0.35 & 0.86 \\
\multirow{2}{*}{ RDKit } & BayesNet & 0.56 & 0.57 & 0.50 & 0.64 \\
& RF-MU & 0.68 & 0.61 & 0.71 & 0.52 \\
& SVM-MU & 0.62 & 0.54 & 0.15 & 0.94 \\
\multirow{2}{*}{ QNA } & BayesNet & 0.63 & 0.62 & 0.63 & 0.60 \\
& RF-DU & 0.50 & 0.58 & 0.25 & 0.89 \\
& SVM-MU & 0.60 & 0.57 & 0.63 & 0.52 \\
\multirow{2}{*}{ Graphs } & GCNN & 0.48 & 0.53 & 0.38 & 0.68 \\
\hline
\end{tabular}

Table S7. In the light of recent advancements in development of novel molecular representations for deep learning, molecular graphs have been claimed to outperform traditional descriptors in predicting chemical properties and biological activities [29, 30]. These studies employed graph features as descriptors to perform QSAR modeling on large number of datasets from different domains using the GCNN architecture. Therefore, we employed the most recent version [29] of this algorithm for prediction of cytosol stability of the validation set compounds. Surprisingly, the GCNN models did not perform better than any classical machine learning algorithm employed in this study.

We then investigated the reason behind the inferior performance of the models in external validation. Principal component analysis (see PCA plots in Additional file 1: Figure S1) revealed that the chemical space of the training set is homogeneous, and the compounds are distinct from the external dataset which was derived from a large library of compounds representing diverse chemotypes. This suggests that most external set compounds do not fall within the applicability domain of the training set used to develop the models, limiting their applicability to a new dataset. We believe that a consensus of the best-performing individual models might improve the prediction performance on the external dataset. Since the goal was not to identify the best QSAR model, we did not investigate further into improving the models reported in this study. The modeling methods and corresponding parameters are listed in Additional file 1: Table S4.

\section{MMP validation}

A total of 10 transforms (7 transforms from human data; 3 transforms from mouse data) were applied on in-house library of compounds. We prioritized testing compound pairs that had the largest difference in predicted stability to identify pairs where the compound on the left is potentially unstable. This would allow evaluation of the ability of the chemical transforms to improve cytosolic stability. A quantitative structure-activity relationship (QSAR) model (see previous section for more details) was employed to predict the cytosolic stability of all compounds on left and 96 compound pairs were prioritized for testing in the metabolic assay. In summary, a total of 7 human transforms (96 compound pairs) and 3 mouse transforms (29 compound pairs) were experimentally validated by determining the $t_{1 / 2}$ of the compounds in the very same cytosol stability assay. The number of compound pairs per transformation and their effects on the in-house library compounds are summarized in Table 5 and representative results are shown in Table 6. The complete validation results containing chemical structures of the $125(96+29)$ compound pairs and their $t_{1 / 2}$ values 
Table 5 A summary of the effects of the MMPs selected for external validation ${ }^{\text {a }}$

\begin{tabular}{|c|c|c|c|c|c|c|}
\hline Transform ID & Fragment (left) & Fragment (right) & $\begin{array}{c}\text { \# Pairs } \\
\text { tested }\end{array}$ & Positive & No shift & Negative \\
\hline $\mathrm{T} 1$ & $\mathrm{R}_{1}=\mathrm{N}$ & $\mathrm{R}_{1}^{\prime}$ & 37 & 9 & 17 & 11 \\
\hline $\mathrm{T} 2$ & $\mathrm{R}_{1}-\mathrm{H}$ & $\mathrm{R}_{1}-\mathrm{NH}_{2}$ & 1 & 1 & 0 & 0 \\
\hline $\mathrm{T} 3$ & & & 38 & 13 & 13 & 12 \\
\hline $\mathrm{T} 4$ & $\equiv \mathrm{N}$ & $\mathrm{R}_{1}-S_{\mathrm{O}}^{\|}-\mathrm{NH}_{2}$ & 2 & 1 & 1 & 0 \\
\hline T5 & & & 2 & 2 & 0 & 0 \\
\hline T6 & & & 6 & 4 & 2 & 0 \\
\hline $\mathrm{T} 7$ & & & 6 & 0 & 5 & 1 \\
\hline $\mathrm{t} 1$ & & & 6 & 2 & 4 & 0 \\
\hline $\mathrm{t} 2$ & & & 6 & 3 & 1 & 2 \\
\hline $\mathrm{t} 3$ & & & 15 & 2 & 2 & 11 \\
\hline
\end{tabular}

a $\mathrm{T}$-transforms are based on human data; $\mathrm{t}$ - transforms are based on mouse data 
Table 6 Compound pairs from the in-house library that exemplify the effects of experimentally validated MMP transforms

\begin{tabular}{|c|c|c|c|c|}
\hline Effect & Compound (left) & Compound (right) & $\begin{array}{c}\mathrm{t}_{1 / 2}[\mathrm{~min}] \\
\quad(\mathrm{left})\end{array}$ & $\begin{array}{c}\mathrm{t}_{1 / 2}[\mathrm{~min}] \\
\text { (right) }\end{array}$ \\
\hline Positive & & & 41.6 & $>120.0$ \\
\hline Positive & & & 56.6 & 113.1 \\
\hline Positive & & & 76.2 & $>120.0$ \\
\hline Positive & & & 80.1 & $>120.0$ \\
\hline Positive & & & 110.7 & $>120.0$ \\
\hline Undetermined & & & $>120.0$ & $>120.0$ \\
\hline Undetermined & & & $>120.0$ & $>120.0$ \\
\hline Undetermined & & & $>120.0$ & $>120.0$ \\
\hline Negative & & & $>120.0$ & 70.2 \\
\hline Negative & & & 62.9 & 36.3 \\
\hline Negative & & & 82.6 & 40.1 \\
\hline Negative & & & 93.0 & 18.5 \\
\hline
\end{tabular}


from the stability assay are provided in the supporting material (Additional file 3). Due to the proprietary nature of the dataset, we could not provide the structures of the training set. However, exemplary transformations are provided in Additional file 1: Tables S2 and S3.

While the human transforms were represented by distinct fragments on both left and right sides, all mouse transforms had the same fragment on the left side. In either case, the number of compound pairs per transform varied, with some representing more than 30 pairs and some representing only one or two pairs of compounds. For instance, the amine substitution represents only one out of the 96 pairs tested although the set of 1166 compound pairs comprises another 45 pairs represented by this transform. The very same transform represents 53 compound pairs in the training set with an average shift of $62.3( \pm 51.4) \mathrm{min}$, which indicates that on average the $t_{1 / 2}$ increased by $62 \mathrm{~min}$. The geometric mean of the shift in $t_{1 / 2}$ was found to be $32 \mathrm{~min}$. Due to the lack of adequate number of validation examples per transform, we did not decide upon explicit criteria to identify significant transforms. Overall, the transforms demonstrated mixed effects. Few transforms resulted in 'positive shift' and 'no shift' while others demonstrated a 'negative shift.'

Out of the 96 compound pairs based on human transforms, two pairs had to be excluded from the analysis due to lack of mass-spectrometry signal. We identified seven pairs from the remaining 94, for which the compound on left-hand side was unstable. Three of these compounds $\left(t_{1 / 2}=8.2,17.4\right.$ and $\left.17.4 \mathrm{~min}\right)$ could be successfully transformed into stable compounds $\left(t_{1 / 2}=35.6,93.0\right.$ and $38.3 \mathrm{~min}$ ). We observed no change in $t_{1 / 2}$ values for remaining four unstable compounds. However, a total of 17 pairs demonstrated 1.5 -fold increase in $t_{1 / 2}$. While, a total of nine pairs from total 96 demonstrated 'significant negative shift', we could not conclude the effect of transforms on 40 pairs since the compounds on both left- and right-hand side demonstrated a $t_{1 / 2}$ value of $>120 \mathrm{~min}$. Since the $t_{1 / 2}$ values could not be extrapolated beyond $120 \mathrm{~min}$, we consider the effect of transforms on these pairs as inconclusive or undetermined.

Only two out of 29 compound pairs based on mouse transforms comprised of an unstable compound on the left-hand side. Of the two, one pair showed a 'positive shift' and the other one showed 'no shift'. Two pairs out of 29 total pairs demonstrated a 'significant negative shift', while seven were inconclusive or undetermined. The $t_{1 / 2}$ improved by more than 1.5-fold only in case of four pairs. In both human and mouse datasets, few compound pairs demonstrated a 'shift' that could not be categorized as either positive or negative, i.e., the $t_{1 / 2}$ values were within the standard error of the assay. The poor performance of mouse transforms could be explained by the greater skewness of the training set towards stable class in comparison to the human dataset. Example compound pairs from the in-house library demonstrating positive or negative effects (based on human transforms) are listed in Table 6. The whole dataset is listed as part of supplemental.

\section{Discussion}

Optimization of different pharmacokinetic and toxicity endpoints is fundamental to efficient drug discovery [34]. CYP-mediated metabolism represents a major route of elimination of many drugs [35-38]. Significant progress has been made in reducing metabolic instabilities due to CYP-mediated oxidation [39]. A majority of these low microsomal turnover compounds could be potential substrates for non-CYP metabolism [38, 40, 41]. Many phase I drug metabolizing enzymes are present in the cytosol including aldehyde oxidase, alcohol dehydrogenase, xanthine oxidase (XO), esterases, amidases/ peptidases and aldo-keto reductases [42]. Since we did not fortify our assay with additional co-factors, majority of our high clearance compounds would be substrates of either $\mathrm{AO}, \mathrm{XO}$, amidases/peptidases or esterases. 7\% of our total dataset and $4 \%$ of substrates $\left(\mathrm{t}_{1 / 2}<60 \mathrm{~min}\right.$ ) contain ester or amide groups (see Table 7). This points toward a major role of $\mathrm{AO}$ - and $\mathrm{XO}$-mediated metabolism in our datasets. AO and XO belong to a family of molybdenum-containing enzymes and these enzymes exhibit an extraordinary degree of amino acid sequence similarity. Since the process of making human cytosol fractions involves the use of allopurinol (a potent XO inhibitor), human cytosol fractions are typically devoid of any XO activity [43]. Thus, it is possible that the majority of human transform rules are derived from substrates of AO. In case of the mouse dataset, it is likely that the transform rules are derived from $\mathrm{AO}$ and $\mathrm{XO}$ substrates.

Matched molecular pairs analysis has been systematically applied in both academia and industry to extract knowledge from small molecule databases with measured properties and bioactivities [14, 34, 44]. We used this computational approach to identify chemical transformations with a potential to affect cytosolic stability. The human dataset provided more transformations as compared to the mouse data. This was contradictory to our expectations since mice have four AO-isozymes

\begin{tabular}{|c|c|c|c|}
\hline Dataset & \# Substrates & $\begin{array}{l}\text { Amide matches (\% } \\
\text { substrates) }\end{array}$ & $\begin{array}{l}\text { Ester matches } \\
\text { (\% substrates) }\end{array}$ \\
\hline Human & 505 & $14(2.8 \%)$ & $11(2.2 \%)$ \\
\hline Mouse & 235 & $8(3.4 \%)$ & $4(1.7 \%)$ \\
\hline
\end{tabular}


compared to only one in humans. We identified that none of the transformations based on mouse data improved cytosolic stability in general, partly due to the skewed nature of the training set (ratio of unstable to stable $\sim 0.1)$. Although some transformations based on human data resulted in a positive shift, these are small numbers when compared to the number of examples available for molecular transformations in the industry catalogs which are derived from large compound collections $[34,45,46]$. Since this was anticipated, our criteria for identifying significant transformations were not too strict. As long as all example pairs show a 'positive shift' from left to right, the transformation was considered valuable. We made an exception in case of those transformations that demonstrated 'no shift' and considered them useful only when they demonstrated a positive shift and no 'negative shift'. Those transformations representing at least as many 'negative shift' examples as the 'positive shift' examples were not considered useful. In case there were more 'no shift' examples compared to the 'negative shift' examples, the transformations were considered neutral. For all transforms resulting in either 'positive shift' or 'no shift', we identified the compound pairs in the training set and examined if the pattern remains the same. The mean $t_{1 / 2}$ values of the compounds from the training set involved in these transformations are listed in Table 8. Although they always demonstrated a positive shift, the limited number of examples impede the chances of obtaining consistent trends. Transformations with at least two compound pairs represent nearly $20 \%$ of the total unique transforms, while those representing 10 or more pairs were only $1 \%$. Therefore, we plan on testing additional compounds as a follow-up to validate the general applicability of these transformations.

Several computational models of metabolic stability are available in the literature. Most of them focused on predicting metabolic stability in human liver microsomes, indicating that these models are predictive of CYP450-mediated metabolism [25, 47-50]. While some experimental and in silico efforts attempted to predict non-CYP450 mediated clearance (e.g., aldehyde oxidase metabolism) of drugs, most dealt with only a handful of compounds [51-55]. Few studies relied on descriptors such as energy of the tetrahedral intermediate and steric hindrance to develop in silico prediction models [52]. Such descriptors cannot be computed in silico for a large number of chemical compounds. Therefore, we focused on generating QSAR models using molecular descriptors that are routinely used in cheminformatics modeling exercises. Furthermore, recent studies [56, 57] reported that QSAR efforts can be augmented with MMPA. In this modeling strategy, the effects of transformations generated using MMPA are subsequently evaluated using QSAR predictions. In the first study, Warner et al. reported the use of aggregation effect of multiple transformations to generate novel compounds with improved predictions as compared to models based on nearest neighbors [58] and random forests [56, 59]. Similarly, Springer et al. [57] reported delta-models trained on MMPs to predict activity change resulting from a particular chemical transformation. More recently, Keefer et al. [46] applied an opposite strategy to evaluate the effect of chemical transformations on lipophilicity and metabolic clearance. Here, they employed QSAR techniques first to predict activities for compounds with no experimental data. Later, MMPA was performed to evaluate the effect of chemical transformations on the basis of predicted activities. We employed the same strategy in a different way. We applied the selected transformations on a large chemical library with diverse compounds and then used a QSAR model to identify pairs that could be tested. To the best of our knowledge, the QSAR models reported in this study are the first in silico models that predict cytosolic stability. We could not identify molecular properties (i.e., MW, TPSA, $\log$ P) that correlate with this pharmacokinetic endpoint of emerging importance, but we reported models based on different sets of molecular descriptors. Further assessment on the validation set compounds revealed that only a fraction of them fall within the applicability domain of the training set compounds. Again, lack of a dataset with structurally diverse compounds hinders development of a robust classifier that should identify compounds with potential cytosol-mediated metabolic liabilities. Therefore, efforts

Table 8 Stability data for the training set compounds involved in 'positive' and 'no shift' transformations

\begin{tabular}{lllll}
\hline Transform ID & \# Training examples & Avg. $\mathbf{t}_{\mathbf{1 / 2}}$ [min] (left) & Avg. $\mathbf{t}_{\mathbf{1 / 2}}$ [min] (right) & $\begin{array}{l}\text { Avg. of } \boldsymbol{\Delta} \\
\mathbf{t}_{\mathbf{1 / 2}} \text { [min] }\end{array}$ \\
\hline T2 & 53 & $20.7 \pm 33.4$ & $83.0 \pm 47.0$ & 32.0 \\
T4 & 3 & $20.8 \pm 9.8$ & $116.2 \pm 6.6$ & 94.9 \\
T5 & 1 & $19.2 \pm 0.0$ & $>120.0$ & $\mathrm{~N} / \mathrm{A}$ \\
T6 & 3 & $24.4 \pm 5.1$ & $>120.0$ & 95.5 \\
T7 & 2 & $4.0 \pm 0.0$ & $96.7 \pm 33.0$ & 89.7 \\
\hline
\end{tabular}

The geometric mean of $\Delta t_{1 / 2}$ is provided in addition to the average of $t_{1 / 2}$ of compounds on left and right 
are needed to generate stability data for a diverse collection of compounds in order to generate generally applicable transforms and improve the models reported in this study.

\section{Conclusion}

Little knowledge exists on how to avoid cytosolic enzyme-mediated metabolism of small molecules in comparison to xenobiotic metabolism by microsomal enzymes such as CYP450s. In this study, we generated structure-metabolic stability data for 1450 compounds in order to derive chemical transformation rules that could help medicinal chemists overcome cytosolic metabolism liabilities. Matched molecular pairs analysis was performed to identify rules that were validated on an independent chemical library. Further experimental validation was performed to assess the validity of these rules. To the best of our knowledge, this study reports the first QSAR models for prediction of cytosolic stability of small molecules. Additionally, we release $t_{1 / 2}$ data for about 250 diverse compounds from an in-house library to encourage the community to further develop and validate models for this increasingly important pharmacokinetic endpoint.

\section{Supplementary information}

Supplementary information accompanies this paper at https://doi. org/10.1186/s13321-020-00426-7.

Additional file 1. Additional figures and tables.

Additional file 2. MMPA KNIME workflow.

Additional file 3. External validation results.

\section{Abbreviations}

2D: Two-dimensional; AO: Aldehyde oxidase; AUC-ROC: Area under the ROC curve; BACC: Balanced accuracy; CYP: Cytochrome P450; FN: False negatives; FP: False positives; MMPA: Matched Molecular Pairs Analysis; MOE: Molecular Operating Environment; MW: Molecular weight; QSAR: Quantitative-structure activity relationship; TPSA: Topological polar surface area; TN: True negatives; TP:True positives; XO: Xanthine oxidase.

\section{Acknowledgements}

The authors would like to acknowledge Drs. Wenwei Huang, Hao Li, Khalida Shamim and Jian-Kang Jiang for helpful discussions.

\section{Authors' contributions}

The manuscript was written through contributions of all authors. All authors have given approval to the final version of the manuscript. All authors read and approved the final manuscript.

\section{Funding}

This research was supported by the Intramural Research Program of the National Institutes of Health, National Center for Advancing Translational Sciences.

\section{Availability of data and materials}

Compounds used in the training dataset come from active projects at NCATS and the data generated from those compounds cannot be made public currently. However, the dataset supporting the conclusions of this article is included in the additional material in a machine-readable format.

\section{Competing interests}

The authors declare that they have no competing interests.

Received: 30 September 2019 Accepted: 25 March 2020

Published online: 07 April 2020

\section{References}

1. Fukami T, Yokoi T (2012) The emerging role of human esterases. Drug Metab Pharmacokinet 27(5):466-477

2. Diamond S, Boer J, Maduskuie TP Jr, Falahatpisheh N, Li Y, Yeleswaram S (2010) Species-specific metabolism of SGX523 by aldehyde oxidase and the toxicological implications. Drug Metab Dispos 38(8):1277-1285

3. Sanoh S, Tayama Y, Sugihara K, Kitamura S, Ohta S (2015) Significance of aldehyde oxidase during drug development: effects on drug metabolism, pharmacokinetics, toxicity, and efficacy. Drug Metab Pharmacokinet 30(1):52-63

4. Akabane $\mathrm{T}$, Tanaka $\mathrm{K}$, Irie M, Terashita S, Teramura $\mathrm{T}$ (2011) Case report of extensive metabolism by aldehyde oxidase in humans: pharmacokinetics and metabolite profile of FK3453 in rats, dogs, and humans. Xenobiotica 41(5):372-384

5. Nassar AE, Kamel AM, Clarimont C (2004) Improving the decision-making process in the structural modification of drug candidates: enhancing metabolic stability. Drug Discov Today 9(23):1020-1028

6. Kerns EH, Di L (2008) Drug-like properties: concepts, structure design and methods. pp 1-528

7. Montefiori M, Jørgensen FS, Olsen L (2017) Aldehyde oxidase: reaction mechanism and prediction of site of metabolism. Acs Omega 2(8):4237-4244

8. O'Hara F, Burns AC, Collins MR, Dalvie D, Ornelas MA, Vaz ADN, Fujiwara Y, Baran PS (2014) A simple litmus test for aldehyde oxidase metabolism of heteroarenes. J Med Chem 57(4):1616-1620

9. Abbasi A, Paragas EM, Joswig-Jones CA, Rodgers JT, Jones JP (2019) Time course of aldehyde oxidase and why it is nonlinear. Drug Metab Dispos 47(5):473

10. Kitamura S, Sugihara K, Nakatani K, Ohta S, Oh-Hara T, Ninomiya S-I, Green CE, Tyson CA (1999) Variation of hepatic methotrexate 7-hydroxylase activity in animals and humans. IUBMB Life 48(6):607-611

11. Kenny PW, Sadowski J (2005) Structure modification in chemical databases. Methods Princ Med Chem 22:271-285

12. Dossetter AG, Griffen EJ, Leach AG (2013) Matched molecular pair analysis in drug discovery. Drug Discov Today 18(15):724-731

13. Griffen E, Leach AG, Robb GR, Warner DJ (2011) Matched molecular pairs as a medicinal chemistry tool. J Med Chem 54(22):7739-7750

14. Leach AG, Jones HD, Cosgrove DA, Kenny PW, Ruston L, MacFaul P, Wood JM, Colclough N, Law B (2006) Matched molecular pairs as a guide in the optimization of pharmaceutical properties; a study of aqueous solubility, plasma protein binding and oral exposure. J Med Chem 49(23):6672-6682

15. Shah P, Kerns E, Nguyen DT, Obach RS, Wang AQ, Zakharov A, McKew J, Simeonov A, Hop CE, Xu X (2016) An automated high-throughput metabolic stability assay using an integrated high-resolution accurate mass method and automated data analysis software. Drug Metab Dispos 44(10):1653-1661

16. Hussain J, Rea C (2010) Computationally efficient algorithm to identify matched molecular pairs (MMPs) in large data sets. J Chem Inf Model 50(3):339-348

17. Berthold MR, Cebron N, Dill F, Gabriel TR, Kotter T, Meinl T, Ohl P, Sieb C, Thiel K, Wiswedel B (2008) KNIME: the Konstanz Information Miner. Stud Class Data Anal. pp 319-326

18. Roughley S (2018) Five years of the KNIME vernalis cheminformatics community contribution. Curr Med Chem. https://doi.org/10.2174/09298 67325666180904113616

19. RDKit: Open-source cheminformatics. http://www.rdkit.org

20. Breiman L (2001) Random forests. Mach Learn 45(1):5-32 
21. Cortes C, Vapnik V (1995) Support-vector networks. Mach Learn 20(3):273-297

22. Heikamp K, Bajorath J (2014) Support vector machines for drug discovery. Expert Opin Drug Discov 9(1):93-104

23. Bouckaert RR (2004) Bayesian network classifiers in weka

24. Varnek A, Baskin I (2012) Machine learning methods for property prediction in chemoinformatics: Quo Vadis? J Chem Inf Model 52(6):1413-1437

25. Zakharov AV, Peach ML, Sitzmann M, Filippov IV, McCartney HJ, Smith LH, Pugliese A, Nicklaus MC (2012) Computational tools and resources for metabolism-related property predictions. 2. Application to prediction of half-life time in human liver microsomes. Future Med Chem 4(15):1933-1944

26. Chemical Computing Group I (2008) MOE (molecular operating environment). Montreal, Canada

27. Lagunin A, Zakharov A, Filimonov D, Poroikov V (2011) QSAR modelling of rat acute toxicity on the basis of PASS prediction. Mol Inform 30(2-3):241-250

28. Filimonov DA, Zakharov AV, Lagunin AA, Poroikov VV (2009) QNA-based 'Star Track' QSAR approach. SAR QSAR Environ Res 20(7-8):679-709

29. Yang K, Swanson K, Jin W, Coley C, Eiden P, Gao H, Guzman-Perez A, Hopper T, Kelley B, Mathea M et al (2019) Analyzing learned molecular representations for property prediction. J Chem Inf Model 59(8):3370-3388

30. Wu Z, Ramsundar B, Feinberg Evan N, Gomes J, Geniesse C, Pappu AS, Leswing K, Pande V (2018) MoleculeNet: a benchmark for molecular machine learning. Chem Sci 9(2):513-530

31. Zakharov AV, Peach ML, Sitzmann M, Nicklaus MC (2014) QSAR modeling of imbalanced high-throughput screening data in PubChem. J Chem Inf Model 54(3):705-712

32. Zhang X, Liu HH, Weller P, Zheng M, Tao W, Wang J, Liao G, Monshouwer M, Peltz G (2011) In silico and in vitro pharmacogenetics: aldehyde oxidase rapidly metabolizes a p38 kinase inhibitor. Pharmacogenomics J 11(1):15-24

33. Kaye B, Rance DJ, Waring L (1985) Oxidative metabolism of carbazeran in vitro by liver cytosol of baboon and man. Xenobiotica 15(3):237-242

34. Keefer CE, Chang G, Kauffman GW (2011) Extraction of tacit knowledge from large ADME data sets via pairwise analysis. Bioorg Med Chem 19(12):3739-3749

35. Lin JH, Lu AY (1998) Inhibition and induction of cytochrome P450 and the clinical implications. Clin Pharmacokinet 35(5):361-390

36. Di $L$ (2014) The role of drug metabolizing enzymes in clearance. Expert Opin Drug Metab Toxicol 10(3):379-393

37. Pryde DC, Dalvie D, Hu Q, Jones P, Obach RS, Tran TD (2010) Aldehyde oxidase: an enzyme of emerging importance in drug discovery. J Med Chem 53(24):8441-8460

38. Argikar UA, Potter PM, Hutzler JM, Marathe PH (2016) Challenges and opportunities with non-CYP enzymes aldehyde oxidase, carboxylesterase, and UDP-glucuronosyltransferase: focus on reaction phenotyping and prediction of human clearance. AAPS J 18(6):1391-1405

39. Dalvie D, Kang P, Loi CM, Goulet L, Nair S (2010) Influence of heteroaromatic rings on ADME properties of drugs. Rsc Drug Discov 1:328-369

40. Garattini E, Terao M (2013) Aldehyde oxidase and its importance in novel drug discovery: present and future challenges. Expert Opin Drug Discov 8(6):641-654

41. Akabane T, Gerst N, Naritomi Y, Masters JN, Tamura K (2012) A practical and direct comparison of intrinsic metabolic clearance of several nonCYP enzyme substrates in freshly isolated and cryopreserved hepatocytes. Drug Metab Pharmacokinet 27(2):181-191

42. Penner N, Woodward C, Prakash C (2012) Drug metabolizing enzymes and biotransformation reactions. In: Zhang D, Austin SS (eds) ADMEenabling technologies in drug design and development. Wiley, New York

43. Barr JT, Choughule KV, Nepal S, Wong T, Chaudhry AS, Joswig-Jones CA, Zientek M, Strom SC, Schuetz EG, Thummel KE et al (2014) Why do most human liver cytosol preparations lack xanthine oxidase activity? Drug Metab Dispos 42(4):695-699

44. Gleeson P, Bravi G, Modi S, Lowe D (2009) ADMET rules of thumb II: a comparison of the effects of common substituents on a range of ADMET parameters. Bioorg Med Chem 17(16):5906-5919

45. Papadatos G, Alkarouri M, Gillet VJ, Willett P, Kadirkamanathan V, Luscombe CN, Bravi G, Richmond NJ, Pickett SD, Hussain J et al (2010) Lead optimization using matched molecular pairs: inclusion of contextual information for enhanced prediction of HERG inhibition, solubility, and lipophilicity. J Chem Inf Model 50(10):1872-1886

46. Koutsoukas A, Chang G, Keefer CE (2019) In-silico extraction of design ideas using MMPA-by-QSAR and its application on ADME endpoints. J Chem Inf Model 59(1):477-485

47. Ekins S (2003) In silico approaches to predicting drug metabolism, toxicology and beyond. Biochem Soc Trans 31(Pt 3):611-614

48. Hu Y, Unwalla R, Denny RA, Bikker J, Di L, Humblet C (2010) Development of QSAR models for microsomal stability: identification of good and bad structural features for rat, human and mouse microsomal stability. J Comput Aided Mol Des 24(1):23-35

49. Lee PH, Cucurull-Sanchez L, Lu J, Du YJ (2007) Development of in silico models for human liver microsomal stability. J Comput Aided Mol Des 21(12):665-673

50. Sakiyama Y, Yuki H, Moriya T, Hattori K, Suzuki M, Shimada K, Honma T (2008) Predicting human liver microsomal stability with machine learning techniques. J Mol Graph Model 26(6):907-915

51. Torres RA, Korzekwa KR, McMasters DR, Fandozzi CM, Jones JP (2007) Use of density functional calculations to predict the regioselectivity of drugs and molecules metabolized by aldehyde oxidase. J Med Chem 50(19):4642-4647

52. Xu Y, Li L, Wang Y, Xing J, Zhou L, Zhong D, Luo X, Jiang H, Chen K, Zheng $M$ et al (2017) Aldehyde oxidase mediated metabolism in drug-like molecules: a combined computational and experimental study. J Med Chem 60(7):2973-2982

53. Jones JP, Korzekwa KR (2013) Predicting intrinsic clearance for drugs and drug candidates metabolized by aldehyde oxidase. Mol Pharm 10(4):1262-1268

54. Dalvie D, Sun H, Xiang C, Hu Q, Jiang Y, Kang P (2012) Effect of structural variation on aldehyde oxidase-catalyzed oxidation of zoniporide. Drug Metab Dispos 40(8):1575-1587

55. Pryde DC, Tran TD, Jones P, Duckworth J, Howard M, Gardner I, Hyland R, Webster R, Wenham T, Bagal S et al (2012) Medicinal chemistry approaches to avoid aldehyde oxidase metabolism. Bioorg Med Chem Lett 22(8):2856-2860

56. Warner DJ, Bridgland-Taylor MH, Sefton CE, Wood DJ (2012) Prospective prediction of antitarget activity by matched molecular pairs analysis. Mol Inform 31(5):365-368

57. Beck JM, Springer C (2014) Quantitative structure-activity relationship models of chemical transformations from matched pairs analyses. J Chem Inf Model 54(4):1226-1234

58. Altman NS (1992) An Introduction to kernel and nearest-neighbor nonparametric regression. Am Stat 46(3):175-185

59. Ho TK (1998) The random subspace method for constructing decision forests. IEEE T Pattern Anal 20(8):832-844

\section{Publisher's Note}

Springer Nature remains neutral with regard to jurisdictional claims in published maps and institutional affiliations. 